\title{
Generalized integral inequalities on time scales
}

Tooba Fayyaz ${ }^{1 *}$, Nazia Irshad ${ }^{1}$, Asif R Khan' , Ghaus ur Rahman² and Gholam Roqia ${ }^{3}$

"Correspondence:

i.ttabi@yahoo.com

'Department of Mathematics,

University of Karachi, University

Road, Karachi, 75270, Pakistan

Full list of author information is

available at the end of the article

\section{Introduction}

In the past few years, time scales calculus has fascinated scientists by virtue of its tremendous application in many branches of sciences, e.g. population dynamics, finance, physics, etc. [1, 2]. During the last decade, with the evolution of integral and differential equations as in including difference equations, voluminous integral and differential inequalities have been disclosed [3-8]. These handy discoveries concern a significant part in the research of boundedness, comprehensive existence, stability of solutions of integral and differential equations, and likewise of difference equations. Subsequently, Hilger [9] put forward the time scales' theory as a theory that combines differential and difference calculus in a sophisticated manner. Afterwards several researchers have talked about various facets of the dynamic equations on time scales including various inequalities in detail $[8,10]$. However, the Feng Qi integral inequalities on time scales have been given limited attention.

In 2000, Feng Qi explored some new types of integral inequalities and presented his results through an analytic approach and mathematical induction. His work motivated many researchers and a group of people at different times continued the work of Feng Qi. Mohamed Akkouchi, [11] studied a general version of a problem posed by Feng Qi [12] in the context of a measured space equipped with a positive finite measure. Hong Yong [13] in his short note introduced some parameters and imposed sufficient conditions so that Qi type integral inequality holds.

Moreover, Kamel Brahim et al. [14] introduced some Feng Qi type $q$-integral inequalities, in quantum calculus.

(c) 2016 Fayyaz et al. This article is distributed under the terms of the Creative Commons Attribution 4.0 International License (http://creativecommons.org/licenses/by/4.0/), which permits unrestricted use, distribution, and reproduction in any medium, provided you give appropriate credit to the original author(s) and the source, provide a link to the Creative Commons license, and indicate if changes were made. 
Motivated by the afore-mentioned discussion, the present article is concerned with Feng Qi integral inequalities on time scales. To the best of the authors' knowledge, no contribution is available in the literature about Feng Qi type integral inequalities on time scales.

\section{Preliminaries}

For the convenience of the reader, we provide some significant and main characteristics of time scale calculus, basic definitions, and results. By a time scale we understand any non-empty and closed subset of real numbers with the ordering inherited from the set of real numbers. Although [2] and [15] are good sources to study the basics of time scale and quantum calculus, we just state some definitions, important results, and notations necessary for our article. Throughout this paper we will denote a time scale by the symbol $\mathbb{T}$ and we mean $[a, b] \cap \mathbb{T}$ by $[a, b]_{\mathbb{T}}$, where $[a, b] \subset \mathbb{R}$. From $[2]$, we have extracted some definitions and results which are as follows.

Definition 2.1 For $u \in \mathbb{T}$ we define the forward jump operator $\sigma: \mathbb{T} \rightarrow \mathbb{T}$ by

$$
\sigma(u):=\inf \{v \in \mathbb{T}: v>u\}
$$

and the backward jump operator $\rho: \mathbb{T} \rightarrow \mathbb{T}$ is defined by

$$
\rho(u):=\sup \{v \in \mathbb{T}: v<u\}
$$

while the graininess function $\mu: \mathbb{T} \rightarrow[0, \infty)$ is defined by

$$
\mu(u):=\sigma(u)-u
$$

and $v: \mathbb{T} \rightarrow[0, \infty)$ is defined as

$$
v(u):=u-\rho(u)
$$

Here we define $\mathbb{T}^{k}, \mathbb{T}_{k}$ as follows:

$$
\mathbb{T}^{k}= \begin{cases}\mathbb{T} \backslash(\rho(\sup \mathbb{T}), \sup \mathbb{T}) & \text { if } \sup \mathbb{T}<\infty \\ \mathbb{T} & \text { if } \sup \mathbb{T}=\infty\end{cases}
$$

and

$$
\mathbb{T}_{k}= \begin{cases}\mathbb{T} \backslash[\inf \mathbb{T}, \sigma(\inf \mathbb{T})) & \text { if } \inf \mathbb{T}>-\infty \\ \mathbb{T} & \text { if } \inf \mathbb{T}=-\infty\end{cases}
$$

Proposition 2.2 Let $\phi: \mathbb{T} \rightarrow \mathbb{R}$ be a continuous function.

(i) If $\sigma(u)>u$, then $\phi$ is $\Delta$-differentiable at $u \in \mathbb{T}^{k}$ with

$$
\phi^{\Delta}(u)=\frac{\phi(\sigma(u))-\phi(u)}{\mu(u)} .
$$


(ii) If $\rho(u)<t$, then $\phi$ is $\nabla$-differentiable at $u \in \mathbb{T}_{k}$ with

$$
\phi^{\nabla}(u)=\frac{\phi(u)-\phi(\rho(u))}{v(u)} .
$$

Proposition 2.3 Assume $\phi, f: \mathbb{T} \rightarrow \mathbb{R}$ are $\Delta$-differentiable at $u \in \mathbb{T}^{k}$. Then:

(i) The sum $\phi+f: \mathbb{T} \rightarrow \mathbb{R}$ is $\Delta$-differentiable at $u$ with

$$
(\phi+f)^{\Delta}(u)=\phi^{\Delta}(u)+f^{\Delta}(u) .
$$

(ii) For any constant $c, c \phi: \mathbb{T} \rightarrow \mathbb{R}$ is $\Delta$-differentiable at $u$ with

$$
(c \phi)^{\Delta}(u)=c \phi^{\Delta}(u)
$$

Proposition 2.4 Assume $\phi, f: \mathbb{T} \rightarrow \mathbb{R}$ are $\nabla$-differentiable at $u \in \mathbb{T}_{k}$. Then:

(i) The sum $\phi+f: \mathbb{T} \rightarrow \mathbb{R}$ is $\nabla$-differentiable at $u$ with

$$
(\phi+f)^{\nabla}(u)=\phi^{\nabla}(u)+f^{\nabla}(u) .
$$

(ii) For any constant $c, c f: \mathbb{T} \rightarrow \mathbb{R}$ is $\nabla$-differentiable at $u$ with

$$
(c f)^{\nabla}(u)=c f^{\nabla}(u)
$$

Proposition 2.5 Assume that $f: \mathbb{T} \rightarrow \mathbb{R}$ is strictly increasing and $\tilde{\mathbb{T}}:=f(\mathbb{T})$ is a time scale. Let $\phi: f(\mathbb{R}) \rightarrow \mathbb{R}$. If $f^{\Delta}$ and $\phi^{\tilde{\Delta}}$ exist for $t \in \mathbb{T}^{k}$ then

$$
(\phi \circ f)^{\Delta}=\left(\phi^{\tilde{\Delta}} \circ f\right) f^{\Delta} .
$$

Proposition 2.6 Every continuous function has an antiderivative. In particular if $u_{0} \in \mathbb{T}$, then the pre-differentiable function $\varphi$ defined by

$$
\varphi(u)=\int_{u_{0}}^{u} \phi(\theta) \Delta \theta \quad \text { for } u \in \mathbb{T}
$$

is an antiderivative of $\phi$.

Proposition 2.7 Let $u_{0} \in \mathbb{T}, v_{0} \in \mathbb{R}$ and $\phi: \mathbb{T}^{k} \rightarrow \mathbb{R}$ be a continuous map. Then there exists a unique pre-differentiable function $\varphi$ satisfying

$$
\varphi^{\Delta}(u)=\phi(u) \quad \text { for all } u \in D, \quad \phi\left(u_{0}\right)=v_{0}
$$

where $D$ is the region of differentiation.

\section{Proposition 2.8}

(i) Assume that $\phi: \mathbb{T} \rightarrow \mathbb{R}$ is $\Delta$-differentiable on $\mathbb{T}^{k}$. Then $\phi$ is $\nabla$-differentiable at $u$ and

$$
\phi^{\nabla}(u)=\phi^{\Delta}(\rho(u))
$$

for $u \in \mathbb{T}_{k}$ such that $\sigma(\rho(u))=u$. 
(ii) Assume that $\phi: \mathbb{T} \rightarrow \mathbb{R}$ is $\nabla$-differentiable on $\mathbb{T}_{k}$, then $\phi$ is $\Delta$-differentiable at $u$ and

$$
\phi^{\nabla}(u)=\phi^{\Delta}(\rho(u))
$$

for $u \in \mathbb{T}^{k}$ such that $\rho(\sigma(u))=u$.

In this article, we are interested in a discrete time scale and the most significant discrete time scales are those originating from arithmetic and geometric sequences of real numbers, namely $\mathbb{T}_{h}^{a}:=\{a+k h, k \in \mathbb{Z}\}, h>0$, and $\mathbb{T}_{q}^{a}:=\left\{a q^{k}, k \in \mathbb{Z}\right\} \cup\{0\}, q>1$. In [16], the two-parameter time scale $\mathbb{T}_{(q, h)}^{a}$ is introduced which generalizes the time scales $\mathbb{T}_{h}^{a}$ and $\mathbb{T}_{q}^{a}$. For a given $a \in \mathbb{R}^{+}$, the two-parameter time scale is defined as

$$
\mathbb{T}_{(q, h)}^{a}:=\left\{a q^{k}+[k]_{q} h, k \in \mathbb{Z}\right\} \cup\left\{\frac{h}{1-q}\right\}, \quad q \geq 1, h \geq 0, q+h>1,
$$

where $[k]_{q}=\frac{1-q^{k}}{1-q}$.

Surely $\mathbb{T}_{(q, h)}^{a}=\mathbb{T}_{q}^{a}, \mathbb{T}_{(q, h)}^{a}=\mathbb{T}_{h}^{a}$ provided $h=0$ and $q=1$, respectively (we put $\frac{h}{1-q}:=-\infty$ ). It can be noted that the time scale $\mathbb{T}_{(q, h)}^{a}$ with $a \in \mathbb{R}^{-}$can be considered quite analogously. For the sake of definiteness, we restrict our consideration to $a>0$. Now, the $(q, h)$-derivative of $\phi: \mathbb{T}_{(q, h)}^{a} \rightarrow \mathbb{R}$ is defined at $v$ by

$$
\Delta_{(q, h)} \phi(v)=\frac{\phi(q v+h)-\phi(v)}{(q-1) v+h}
$$

and

$$
\nabla_{(q, h)} \phi(x)=\frac{\phi(v)-\phi\left(q^{-1}(v-h)\right)}{q^{-1}((q-1) v-h)} .
$$

Let $v \in \mathbb{T}_{(q, h)}^{a}$ and let $v>v_{0}$, i.e. there exists $n \in \mathbb{Z}^{+}$such that $v=v_{0} q^{n}+[n]_{q} h$, then we define the delta $(q, h)$-integral by

$$
\int_{v_{0}}^{v} \phi(u) \Delta_{(q, h)} u=\left((q-1) v_{0}+h\right) \sum_{k=0}^{n-1} q^{k} f\left(v_{0} q^{k}+[k]_{q} h\right) .
$$

If we consider the time scale $\mathbb{T}_{(q, h)}^{a}$ with $v_{0}$ replaced by $v$, then the integration over $\left[\frac{h}{1-q}, t\right]$ yields the definition of the nabla $(q, h)$-integral in the form

$$
\int_{h /(1-q)}^{v} \phi(u) \nabla_{(q, h)} u=\left(\left(1-q^{-1}\right) v+q^{-1} h\right) \sum_{k=0}^{\infty} q^{-k} \phi\left(q^{-k} v+[-k]_{q} h\right),
$$

provided that the infinite series converges. More generally, we can consider the nabla $(q, h)$-integral with the lower limit $h /(1-q)$ replaced by $a \in \mathbb{T}_{(q, h)}^{a}, h /(1-q) \leq a<t$. If $h /(1-q)<a$, then the infinite series occurring in (1) is reduced to the corresponding finite series. These definitions extend standard integral introductions: in particular, if $h=0$, then (1) becomes the Jackson $q$-integral [16].

This article has been organized into five sections. In the first section we give an introduction of the article, the second section contains preliminaries which will be used in the 
sequel, and the third section is reserved for results related to Fenq Qi type $\Delta$-integral inequalities defined on a discrete time scale, which generalizes some results given in [17] and [14]. In [17] and [14], there are given the $q$-analog and the $h$-analog, respectively, of some Feng Qi inequalities given in [18, 19], and [20]; therefore, the fourth section will explore the results related to Feng Qi type $\nabla$-integral inequalities defined on a discrete time scale. The last section highlights the important results of present article.

\section{Feng Qi type $\Delta$-integral inequalities}

This section is devoted to the results related to Fenq Qi type $\Delta$-integral inequalities defined on discrete time scales, which generalize some results given in [17] and [14].

Lemma 3.1 Suppose $p \geq 1$ and $f$ is a non-negative and monotonic function on $[a, b]_{\mathbb{T}}$. Then

$$
p f^{p-1}(v) f^{\Delta}(v) \leq\left(f^{p}(v)\right)^{\Delta} \leq p f^{p-1}(\sigma(v)) f^{\Delta}(v)
$$

Proof The definition of the $\Delta$-derivative may be quoted from [16] in the following way:

$$
\left(f^{p}(v)\right)^{\Delta}=\frac{f^{p}(\sigma(v))-f^{p}(v)}{\mu(v)}=\frac{p}{\mu(v)} \int_{f(v)}^{f(\sigma(v))} u^{p-1} d u
$$

Since $f$ is a monotonic increasing function, we obtain

$$
\begin{aligned}
& f^{p-1}(v)[f(\sigma(v))-f(v)] \leq \int_{f(v)}^{f(\sigma(v))} u^{p-1} d u \leq f^{p-1}(\sigma(v))[f(\sigma(v))-f(v)] \\
& p f^{p-1}(v) \frac{f(\sigma(v))-f(v)}{\mu(v)} \leq\left(f^{p}(v)\right)^{\Delta} \leq p f^{p-1}(\sigma(v)) \frac{f(\sigma(v))-f(v)}{\mu(v)} \\
& p f^{p-1}(v) f^{\Delta}(v) \leq\left(f^{p}(v)\right)^{\Delta} \leq p f^{p-1}(\sigma(v)) f^{\Delta}(v) .
\end{aligned}
$$

Remark 3.2 If we set $\sigma(v)=q v+h$ in the preceding lemma defined on $\mathbb{T}_{(q, h)}^{a}$, then we get the following result.

Suppose $p \geq 1$ and $f$ is a non-negative and monotonic function on $[a, b] \cap \mathbb{T}_{(q, h)}^{a}$. Then

$$
p f^{p-1}(v) \Delta_{(q, h)} f(v) \leq \Delta_{(q, h)} f^{p}(v) \leq p f^{p-1}(q v+h) \Delta_{(q, h)} f(v) .
$$

Here, we observe that if we set $q=1$ in this result, we get Lemma 2.1 of [14] and if we set $h=0$ in this result, we get Lemma 2.1 of [17].

Theorem 3.3 Let $\phi$ be a function defined on $[a, b]_{\mathbb{T}}$ such that $\phi(v) \geq 0$ and

$$
\phi^{p-2}(v) \phi^{\Delta}(v) \geq \sigma^{\Delta}(v)(p-2)\left(\sigma^{2}(v)-a\right)^{p-3} \phi^{p-2}\left(\sigma^{2}(v)\right)
$$

$\forall v \in[a, b]_{\mathbb{T}}$ and $p \geq 3$. Then

$$
\int_{a}^{b} \phi^{p}(v) \Delta v \geq\left(\int_{a}^{b} \phi(v) \Delta v\right)^{p-1}
$$


Proof For $v \in[a, b]_{\mathbb{T}}$, we consider the difference

$$
F(v)=\int_{a}^{v} \phi^{p}(u) \Delta u-\left(\int_{a}^{v} \phi(u) \Delta u\right)^{p-1}
$$

by setting

$$
f(v)=\int_{a}^{v} \phi(u) \Delta u
$$

and by using Lemma 3.1, we have

$$
\begin{aligned}
F^{\Delta}(v) & =\phi^{p}(v)-\left(f^{p-1}(v)\right)^{\Delta} \\
& \geq \phi^{p}(v)-(p-1) f^{p-2}(\sigma(v)) f^{\Delta}(v) \\
& =\phi^{p}(v)-(p-1) f^{p-2}(\sigma(v)) \phi(v) \\
& =\phi(v) F_{1}(v),
\end{aligned}
$$

where $F_{1}(v)=\phi^{p-1}(v)-(p-1) f^{p-2}(\sigma(v)) \geq 0$.

Now, again using Lemma 3.1 we get

$$
\begin{aligned}
F_{1}^{\Delta}(v) & =\left(\phi^{p-1}(v)\right)^{\Delta}-(p-1)\left(f^{p-2}(\sigma(v))\right)^{\Delta} \\
& \geq(p-1) \phi^{p-2}(v) \phi^{\Delta}(v)-(p-1)(p-2) f^{p-3}\left(\sigma^{2}(v)\right)(f(\sigma(v)))^{\Delta} \\
& =(p-1) \phi^{p-2}(v) \phi^{\Delta}(v)-(p-1)(p-2) f^{p-3}\left(\sigma^{2}(v)\right) \phi(\sigma(v)) \sigma^{\Delta}(v) .
\end{aligned}
$$

Since the function $\phi$ increases, we have

$$
f\left(\sigma^{2}(v)\right)=\int_{a}^{\sigma^{2}(v)} \phi(u) \Delta u \leq\left(\sigma^{2}(v)-a\right) \phi\left(\sigma^{2}(v)\right)
$$

and therefore

$$
\begin{aligned}
F_{1}^{\Delta}(v) & \geq(p-1)\left[\phi^{p-2}(v) \phi^{\Delta}(v)-\sigma^{\Delta}(v)(p-2)\left(\sigma^{2}(v)-a\right)^{p-3} \phi^{t-3}\left(\sigma^{2}(v)\right) \phi(\sigma(v))\right] \\
& \geq(p-1)\left[\phi^{p-2}(v) \phi^{\Delta}(v)-\sigma^{\Delta}(v)(p-2)\left(\sigma^{2}(v)-a\right)^{p-3} \phi^{p-2}\left(\sigma^{2}(v)\right)\right] \geq 0 .
\end{aligned}
$$

So, we conclude that the function $F_{1}(v)$ increases on $[a, b]_{\mathbb{T}}$. Therefore, $F_{1}(v) \geq F_{1}(a) \geq 0$, which shows the non-negativity of $F^{\Delta}(v)$. Hence, we conclude that $F$ is increasing and $F(v) \geq 0$, so we obtain the required result.

If we choose $\sigma(v)=q v+h$ defined on $\mathbb{T}_{(q, h)}^{a}$ then we get the following corollary.

Corollary 3.4 Let $\phi$ be a function defined on $[a, b] \cap \mathbb{T}_{(q, h)}^{a}$ such that $\phi(v) \geq 0$ and

$$
\phi^{p-2}(v) \Delta_{(q, h)} \phi(v) \geq q(p-2)\left(q^{2} v+[2]_{q} h-a\right)^{p-3} \phi^{p-2}\left(q^{2} v+[2]_{q} h\right)
$$


$\forall v \in[a, b]_{\mathbb{T}}$ and $p \geq 3$. Then

$$
\int_{a}^{b} \phi^{p}(v) \Delta_{(q, h)} v \geq\left(\int_{a}^{b} \phi(v) \Delta_{(q, h)} v\right)^{p-1}
$$

Remark 3.5 Observation shows that Theorem 3 of [17] is a special case of Corollary 3.4 if we simply set $q=1$. Further we note that our result is also a refinement of Proposition 3.2 of [14], and if we set $h=0$ in the above theorem then we get a more refined result as compared to Proposition 3.2 of [14].

Theorem 3.6 If $\phi$ is a non-negative function defined on $[a, b]_{\mathbb{T}}$ such that $\phi(v) \geq 0$ and

$$
\phi^{t}(v) \phi^{\Delta}(v) \geq \frac{\alpha \sigma^{\Delta}(v)\left(\sigma^{2}(v)-a\right)^{\alpha-1}}{(b-a)^{\alpha-1}} \phi^{\alpha}\left(\sigma^{2}(v)\right)
$$

for $v \in[a, b]_{\mathbb{T}}$ and $\alpha \geq 1$, then

$$
\int_{a}^{b} \phi^{\alpha+2}(v) \Delta v \geq \frac{1}{(b-a)^{\alpha-1}}\left(\int_{a}^{b} \phi(v) \Delta v\right)^{\alpha+1} .
$$

Proof We define the following difference, for $v \in[a, b]_{\mathbb{T}}$ :

$$
F(v)=\int_{a}^{v} \phi^{\alpha+2}(v) \Delta v-\frac{1}{(b-a)^{\alpha-1}}\left(\int_{a}^{v} \phi(v) \Delta v\right)^{\alpha+1}
$$

and assume

$$
f(v)=\int_{a}^{v} \phi(u) \Delta u .
$$

Using Lemma 3.1 shows

$$
\begin{aligned}
F^{\Delta}(v) & =\phi^{\alpha+2}(v)-\frac{1}{(b-a)^{\alpha-1}}\left(f^{\alpha+1}(v)\right)^{\Delta} \\
& \geq \phi^{\alpha+2}(v)-\frac{\alpha+1}{(b-a)^{\alpha-1}} f^{\alpha}(\sigma(v)) f^{\Delta}(v) \\
& =\phi^{\alpha+2}(v)-\frac{\alpha+1}{(b-a)^{\alpha-1}} f^{\alpha}(\sigma(v)) \phi(v) \\
& =\phi(v) F_{1}(v)
\end{aligned}
$$

where $F_{1}(v)=\phi^{\alpha+1}(v)-\frac{\alpha+1}{(b-a)^{\alpha-1}} f^{\alpha}(\sigma(v))$.

Now, again by using Lemma 3.1 we obtain

$$
\begin{aligned}
F_{1}^{\Delta}(v) & =\left(\phi^{\alpha+1}(v)\right)^{\Delta}-\frac{\alpha+1}{(b-a)^{\alpha-1}}\left(f^{\alpha}(\sigma(v))\right)^{\Delta} \\
& \geq(\alpha+1) \phi^{\alpha}(v) \phi^{\Delta}(v)-\frac{\alpha(\alpha+1)}{(b-a)^{\alpha-1}} f^{\alpha-1}\left(\sigma^{2}(v)\right)(f(\sigma(v)))^{\Delta} \\
& =(\alpha+1)\left[\phi^{\alpha}(v) \phi^{\Delta}(v)-\frac{\alpha}{(b-a)^{\alpha-1}} f^{\alpha-1}\left(\sigma^{2}(v)\right) \phi(\sigma(v)) \sigma^{\Delta}(v)\right] .
\end{aligned}
$$


Since $\phi$ increases on $[a, b]_{\mathbb{T}}$, for $v \in[a, b]_{\mathbb{T}}$, we have

$$
f\left(\sigma^{2}(v)\right)=\int_{a}^{\sigma^{2}(v)} \phi(u) \Delta u \leq\left(\sigma^{2}(v)-a\right) \phi\left(\sigma^{2}(v)\right)
$$

And therefore,

$$
\begin{aligned}
F_{1}^{\Delta}(v) & \geq(\alpha+1)\left[\phi^{\alpha}(v) \phi^{\Delta}(v)-\alpha \sigma^{\Delta}(v)\left(\frac{\sigma^{2}(v)-a}{b-a}\right)^{\alpha-1} \phi^{\alpha-1}\left(\sigma^{2}(v)\right) \phi(\sigma(v))\right] \\
& \geq(\alpha+1)\left[\phi^{\alpha}(v) \phi^{\Delta}(v)-\alpha \sigma^{\Delta}(v)\left(\frac{\sigma^{2}(v)-a}{b-a}\right)^{\alpha-1} \phi^{\alpha}\left(\sigma^{2}(v)\right)\right] \geq 0 .
\end{aligned}
$$

So, $F_{1}(v)$ is an increasing function. Therefore, $F_{1}(v) \geq F_{1}(a) \geq 0$, which shows the nonnegativity of $F^{\Delta}(v)$. So, we conclude that $F$ is increasing and in addition to $F(v) \geq 0$, hence we get the required result.

We end this section with a corollary and some remarks.

By setting $\sigma(v)=q v+h$ defined on $\mathbb{T}_{(q, h)}^{a}$, then we get the following corollary.

Corollary 3.7 If $\phi$ is a non-negative function defined on $[a, b] \cap \mathbb{T}_{(q, h)}^{a}$ such that $\phi(v) \geq 0$ and

$$
\phi^{\alpha}(v) \Delta_{(q, h)} \phi(v) \geq \frac{\alpha q\left(q^{2} v+[2]_{q} h-a\right)^{\alpha-1}}{(b-a)^{\alpha-1}} \phi^{\alpha}\left(q^{2} v+[2]_{q} h\right)
$$

for $v \in[a, b]_{\mathbb{T}}$ and $\alpha \geq 1$, then

$$
\int_{a}^{b} \phi^{\alpha+2}(v) \Delta_{(q, h)} v \geq \frac{1}{(b-a)^{\alpha-1}}\left(\int_{a}^{b} \phi(v) \Delta_{(q, h)} v\right)^{\alpha+1}
$$

Remark 3.8 It is worth noting that Theorem 4 of [17] becomes a special case of Corollary 3.7 if we simply set $q=1$. Furthermore, it can be noted that this result is a refinement of Proposition 3.5 of [14] and if we set $h=0$ in the above corollary then we get a more refined result than Proposition 3.5 of [14].

\section{Feng Qi type $\nabla$-integral inequalities}

In [17] and [14] there are given the $q$-analog and the $h$-analog, respectively, of some Feng Qi $\nabla$-integral inequalities, which are given in $[18,19]$, and [20]. Hence, this part of the article is concerned with the results related to Fenq Qi type $\nabla$-integral inequalities defined on a discrete time scale.

Lemma 4.1 Suppose $p \geq 1$ is a real number and $f$ be a non-negative and monotonic function on $[a, b]_{\mathbb{T}}$, then

$$
p f^{p-1}(\rho(v)) f^{\nabla}(v) \leq\left(f^{p}(v)\right)^{\nabla} \leq p f^{p-1}(v) f^{\nabla}(v) .
$$

Proof The definition of the $\nabla$-derivative may be quoted from [16] in the following way:

$$
\left(f^{p}(v)\right)^{\nabla}=\frac{f^{p}(v)-f^{p}(\rho(v))}{v(v)}=\frac{p}{v(v)} \int_{f(\rho(v))}^{f(v)} t^{p-1} d t
$$


Since $f$ is an increasing function, we get

$$
\begin{aligned}
& f^{p-1}(\rho(v))[f(v)-f(\rho(v))] \leq \int_{f(\rho(v))}^{f(v)} t^{p-1} d t \leq f^{p-1}(v)[f(v)-f(\rho(v))] \\
& p f^{p-1}(\rho(v)) \frac{f(v)-f(\rho(v))}{v(v)} \leq\left(f^{p}(v)\right)^{\nabla} \leq p f^{p-1}(v) \frac{f(v)-f(\rho(v))}{v(v)}, \\
& p f^{p-1}(\rho(v)) f^{\nabla}(v) \leq\left(f^{p}(v)\right)^{\nabla} \leq p f^{p-1}(v) f^{\nabla}(v) .
\end{aligned}
$$

Remark 4.2 If in the preceding lemma we set $\rho(v)=q^{-1}(v-h)$ defined on $\mathbb{T}_{(q, h)}^{a}$, then we obtain the following result.

Suppose $p \geq 1$ is a real number and $f$ is a non-negative and monotonic function on $[a, b] \cap \mathbb{T}_{(q, h)}^{a}$, then

$$
p f^{p-1}\left(q^{-1}(v-h)\right) \nabla_{(q, h)} f(v) \leq \nabla_{(q, h)} f^{p}(v) \leq p f^{p-1}(v) \nabla_{(q, h)} f(v)
$$

Theorem 4.3 Suppose that $\phi$ is a function defined on $[a, b]_{\mathbb{T}}$ satisfying the condition $\phi(v) \geq$ 0 and

$$
\phi^{\nabla}(v) \geq(p-2)(v-a)^{p-3}
$$

for $v \in[a, b]_{\mathbb{T}}$ and $p \geq 3$. Then

$$
\int_{a}^{b} \phi^{p}(v) \nabla v \geq\left(\int_{a}^{b} \phi(v) \nabla v\right)^{p-1} .
$$

Proof For $v \in[a, b]_{\mathbb{T}}$, we consider the difference

$$
F(v)=\int_{a}^{v} \phi^{p}(u) \nabla u-\left(\int_{a}^{v} \phi(u) \nabla u\right)^{p-1}
$$

and we define

$$
f(v)=\int_{a}^{v} \phi(u) \nabla u .
$$

Now by using Lemma 4.1, we have

$$
\begin{aligned}
F^{\nabla}(v) & =\phi^{p}(v)-\left(f^{p-1}(v)\right)^{\nabla} \\
& \geq \phi^{p}(v)-(p-1) g^{p-2}(v) f^{\nabla}(v) \\
& =\phi^{p}(v)-(p-1) f^{p-2}(v) \phi(v) \\
& =\phi(v) F_{1}(v),
\end{aligned}
$$

where $F_{1}(v)=\phi^{p-1}(v)-(p-1) f^{p-2}(v) \geq 0$.

Now, by virtue of Lemma 4.1 we get

$$
\begin{aligned}
F_{1}^{\nabla}(v) & =\left(\phi^{p-1}(v)\right)^{\nabla}-(p-1)\left(f^{p-2}(v)\right)^{\nabla} \\
& \geq(p-1) \phi^{p-2}(v) \phi^{\nabla}(v)-(p-1)(p-2) f^{p-3}(v) f^{\nabla}(v) \\
& =(p-1) \phi^{p-2}(v) \phi^{\nabla}(v)-(p-1)(p-2) f^{p-3}(v) \phi(v) .
\end{aligned}
$$


Since $\phi$ increases on $[a, b]_{\mathbb{T}}$, for $v \in[a, b]_{\mathbb{T}}$, we have

$$
f(v)=\int_{a}^{v} \phi(u) \nabla u \leq(v-a) \phi(v)
$$

and hence we get

$$
\begin{aligned}
F_{1}^{\nabla}(v) & \geq(p-1)\left[\phi^{p-2}(v) \phi^{\nabla}(v)-(p-2)(v-a)^{p-3} \phi^{p-3}(v) f(v)\right] \\
& \geq(p-1) \phi^{p-2}(v)\left[\phi^{\nabla}(v)-(p-2)(v-a)^{p-3}\right] \geq 0 .
\end{aligned}
$$

So, $F_{1}(v)$ is an increasing function. Therefore, $F_{1}(v) \geq F_{1}(a) \geq 0$, which shows the nonnegativity of $F^{\nabla}(v)$. So, we conclude that $F$ is increasing and $F(v) \geq 0$, hence the assertion follows.

If we choose $\rho(v)=q^{-1}(v-h)$ defined on $\mathbb{T}_{(q, h)}^{a}$, then we get the following corollary.

Corollary 4.4 Suppose that $\phi$ be a function defined on $[a, b] \cap \mathbb{T}_{(q, h)}^{a}$ satisfies the condition $\phi(v) \geq 0$ and

$$
\phi^{\nabla(q, h)}(v) \geq(p-2)(v-a)^{p-3}
$$

for $v \in[a, b]_{\mathbb{T}}$ and $p \geq 3$. Then

$$
\int_{a}^{b} \phi^{p}(v) \nabla_{(q, h)} v \geq\left(\int_{a}^{b} \phi(v) \nabla_{(q, h)} v\right)^{p-1}
$$

Theorem 4.5 Suppose that $\phi$ is a function defined on $[a, b]_{\mathbb{T}}$ satisfying the condition $\phi(v) \geq$ 0 and

$$
\phi^{\nabla}(v) \geq t\left(\frac{v-a}{b-a}\right)^{\alpha-1}
$$

for $v \in[a, b]_{\mathbb{T}}$ and $\alpha \geq 1$, then

$$
\int_{a}^{b} \phi^{\alpha+2}(v) \nabla v \geq \frac{1}{(b-a)^{\alpha-1}}\left(\int_{a}^{b} \phi(v) \nabla v\right)^{\alpha+1}
$$

Proof Assume the following difference, for $v \in[a, b]_{\mathbb{T}}$ :

$$
F(v)=\int_{a}^{v} \phi^{\alpha+2}(v) \nabla v-\frac{1}{(b-a)^{\alpha-1}}\left(\int_{a}^{v} \phi(v) \nabla v\right)^{\alpha+1} .
$$

Also we define the function $f$,

$$
f(v)=\int_{a}^{v} \phi(u) \nabla u
$$


Now by using Lemma 4.1, we have

$$
\begin{aligned}
F^{\nabla}(v) & =\phi^{\alpha+2}(v)-\frac{1}{(b-a)^{\alpha-1}}\left(f^{\alpha+1}(v)\right)^{\nabla} \\
& \geq \phi^{\alpha+2}(v)-\frac{\alpha+1}{(b-a)^{\alpha-1}} f^{\alpha}(v) f^{\nabla}(v) \\
& =\phi^{\alpha+2}(v)-\frac{\alpha+1}{(b-a)^{\alpha-1}} f^{\alpha}(v) \phi(v) \\
& =\phi(v) F_{1}(v),
\end{aligned}
$$

where $F_{1}(v)=\phi^{\alpha+1}(v)-\frac{\alpha+1}{(b-a)^{\alpha-1}} f^{\alpha}(v) \geq 0$.

Again using Lemma 4.1 we get

$$
\begin{aligned}
F_{1}^{\nabla}(v) & =\left(\phi^{\alpha+1}(v)\right)^{\nabla}-\frac{\alpha+1}{(b-a)^{\alpha-1}}\left(f^{\alpha}(v)\right)^{\nabla} \\
& \geq(\alpha+1) \phi^{\alpha}(v) \phi^{\nabla}(v)-\frac{\alpha(\alpha+1)}{(b-a)^{\alpha-1}} f^{\alpha-1}(v) g^{\nabla}(v) \\
& =(\alpha+1) \phi^{\alpha}(v) \phi^{\nabla}(v)-\frac{\alpha(\alpha+1)}{(b-a)^{\alpha-1}} f^{\alpha-1}(v) \phi(v) .
\end{aligned}
$$

Since $\phi$ increases on $[a, b]_{\mathbb{T}}$, for $v \in[a, b]_{\mathbb{T}}$, we have

$$
f(v)=\int_{a}^{v} \phi(u) \nabla u \leq(v-a) \phi(v)
$$

and hence we obtain

$$
\begin{aligned}
F_{1}^{\nabla}(v) & \geq(\alpha+1)\left[\phi^{\alpha}(v) \phi^{\nabla}(v)-\frac{\alpha(v-a)^{\alpha-1}}{(b-a)^{\alpha-1}} \phi^{\alpha-1}(v) \phi(v)\right] \\
& \geq(\alpha+1) \phi^{t}(v)\left[\phi^{\nabla}(v)-\alpha\left(\frac{v-a}{b-a}\right)^{\alpha-1}\right] \geq 0 .
\end{aligned}
$$

Consequently, $F_{1}(v)$ is an increasing function. Therefore, $F_{1}(v) \geq F_{1}(a) \geq 0$, which shows the non-negativity of $F^{\nabla}(v)$. It is also inferred that $F$ is increasing and since $F(v) \geq 0$ we get the required result.

If we choose $\rho(v)=q^{-1}(v-h)$ defined on $\mathbb{T}_{(q, h)}^{a}$ then we get the following corollary.

Corollary 4.6 Let $\phi$ be a function defined on $[a, b] \cap \mathbb{T}_{(q, h)}^{a}$ such that $\phi(v) \geq 0$ and

$$
\phi^{\nabla}(q, h)(v) \geq \alpha\left(\frac{v-a}{b-a}\right)^{\alpha-1}
$$

for $v \in[a, b]_{\mathbb{T}}$ and $\alpha \geq 1$. Then

$$
\int_{a}^{b} \phi^{\alpha+2}(v) \nabla_{(q, h)} v \geq \frac{1}{(b-a)^{\alpha-1}}\left(\int_{a}^{b} \phi(v) \nabla_{(q, h)} v\right)^{\alpha+1}
$$




\section{Conclusion}

The article is aimed to explore Feng Qi integral inequalities on time scales while considering $\Delta$ - and $\nabla$-integrals. The present article generalizes some results of various articles, Lemma 2.1 of $[14,17]$ is a special case of Lemma 3.1. Moreover, observations show that Theorem 3 of [17] is a special case of Corollary 3.4, in addition to results of the present article there is a refinement of Proposition 3.2 in [14]. It can also be noted that Corollary 3.7 gives us Theorem 4 of [14] as a particular case and refines Proposition 3.5 of [17].

Competing interests

The authors declare that they have no competing interests.

\section{Authors' contributions}

ARK and GR made the main contribution in conceiving the presented research. TF, NI, and ARK worked jointly on the second, on the third, and on the fourth sections, while ARK drafted these sections. GUR has drafted and worked solely on the abstract, the Introduction section and the last section and proof-read the whole article. It should be noted that the authors' names are in alphabetical/dictionary order. All authors read and approved the final manuscript.

\section{Author details}

'Department of Mathematics, University of Karachi, University Road, Karachi, 75270, Pakistan. ${ }^{2}$ Department of Mathematics and Statistics, University of Swat, Khyber Pakhtunkhwa, Pakistan. ${ }^{3}$ Comsats Institute of Information Technology, Abbot Abad, Pakistan.

Received: 4 March 2016 Accepted: 7 September 2016 Published online: 26 September 2016

\section{References}

1. Berger, $\mathrm{H}$, Henrich, S, Jessee, J: Application of time scales calculus to the growth and development in populations of Stomoxys calcitrans (Diptera: Muscidae). Int. J. Difference Equ. 8(2), 125-134 (2013)

2. Bohner, M, Peterson, A: Dynamic Equations on Time Scale. Birkhäuser, Boston (2001)

3. Dragomir, SS: On the Hadamard's inequality for convex on the co-ordinates in a rectangle from the plane. Taiwan. J. Math. 5(4), 775-788 (2001)

4. Hsu, K-C: Refinements of Hermite-Hadamard type inequalities for differential co-ordinated convex functions and applications. Taiwan. J. Math. 19(1), 133-157 (2015)

5. Tseng, K-L, Yang, G-S, Hsu, K-C: On some inequalities of Hadamard's type and applications. Taiwan. J. Math. 13(6B), 1929-1948 (2009)

6. Yang, G-S, Tseng, K-L: Inequalities of Hermite-Hadamard-Fejér type for convex functions and Lipschitzian functions. Taiwan. J. Math. 7(3), 433-440 (2003)

7. Wang, D-B, Guan, W: Multiple positive solutions for $p$-Laplacian functional dynamic equations on time scales. Taiwan. J. Math. 12(9), 2327-2340 (2008)

8. Wong, F-H, Lian, W-C, Yu, S-L, Yeh, C-C: Some generalizations of Opial inequalities on time scales. Taiwan. J. Math. 12(2), 463-471 (2008)

9. Hilger, S: Ein Maßkettenkalkül mit Anwendung auf Zentrumsmanning-faltigkeiten. PhD thesis

10. Agarwal, RP, Bohner, M, Peterson, A: Inequalities on time scales: a survey. Math. Inequal. Appl. 4, 535-557 (2001)

11. Akkouchi, M: On an integral inequality of Feng Qi. Divulg. Mat. 13(1), 11-19 (2005)

12. Qi, F: Several integral inequalities. JIPAM. J. Inequal. Pure Appl. Math. 1(2), Article 19 (2000)

13. Yong, H: A note on Feng Qi type integral inequalities. Int. J. Math. Anal. 1(25), 1243-1247 (2007)

14. Brahim, K, Bettaibi, N, Sellemi, M: On some Feng Qi type q-integral inequalities. J. Inequal. Pure Appl. Math. 9(2), Article 43 (2008)

15. Kac, VG, Cheung, P: Quantum Calculus. Universitext. Springer, New York (2002)

16. Čermák, J, Nechvátal, L: On (q,h)-analogue of fractional calculus. J. Nonlinear Math. Phys. 17(1), 51-68 (2010)

17. Krasniqi, V, Shabani, AS: On some Feng Qi type $h$-integral inequalities. Int. J. Open Probl. Comput. Sci. Math. 2(4), 516-521 (2000)

18. Bougoffa, L: Notes on Qi type inequalities. J. Inequal. Pure Appl. Math. 4(4), Article 77 (2003)

19. Mozouzi, S, Qi, F: On an open problem regarding an integral inequality. J. Inequal. Pure Appl. Math. 4(2), Article 31 (2003)

20. Pogany, TK: On an open problem of F. Qi. J. Inequal. Pure Appl. Math. 3(4), Article 54 (2002) 